\title{
Valproic Acid in Prevention and Treatment of COVID-19
}

\author{
Shweta Singh ${ }^{1}$ and Krishna Singh ${ }^{1}$ \\ ${ }^{1}$ Western University
}

May 12, 2020

\begin{abstract}
The novel coronavirus severe acute respiratory syndrome coronavirus-2 (SARS-CoV-2) has posed a serious threat to global public health. Respiratory failure followed by cardiovascular complications with wide-spread endothelial dysfunction and inflammation is rapidly emerging as a key threat in COVID-19. ACE-2 receptors are the cell-entry gate for SARS-CoV-2. Valproic Acid (VPA) is a proposed potential drug to treat COVID-19 but its mechanism of action is not well understood. We demonstrate that VPA-treatment significantly reduced ACE-2 expression in endothelial cells. VPA-treatment significantly reduced the expression of inflammatory cytokines IL-6 along with the endothelial activation marker ICAM-1. We provide evidence and discuss the plausible mechanism in detail for VPA use to prevent and treat COVID-19 in a personalized manner. Our study is expected to entice the scientific and clinical society to investigate VPA as a potential therapeutic option against COVID-19.
\end{abstract}

\section{Introduction}

The pandemic of coronavirus disease 2019 (COVID-19) caused by novel coronavirus severe acute respiratory syndrome coronavirus-2 (SARS-CoV-2) has posed a serious social and economic threat world-wide by infecting over 4 million and killing 276,000 people. The real course of the disease is still not well described but mainly respiratory failure followed by cardiovascular complications with underlying inflammation and thrombus formation are rapidly emerging key threats in COVID-19.[1, 2] A "cytokine storm" or overproduction of pro-inflammatory cytokines such as interleukins (ILs) and tumor necrosis factors (TNFs) is reported in the lungs of COVID-19 patients.[3] Currently, there are no approved drugs or vaccines with proven clinical efficacy to treat or prevent COVID-19, however, the US Food and Drug Administration (FDA) approved limited emergency use for hydroxychloroquine (HCQ). [4, 5] Other drugs such as arbidol, remdesivir, and favipiravir are currently under clinical trial to treat COVID-19 with mixed reports on the efficacy of these drugs in the treatment of COVID-19.[5] There is an urgent need for an effective drug to treat and prevent COVID-19, with minimal side effects.

Limited knowledge about mechanism of infection/action of SARS-CoV-2 appears to be the major problem in identification of therapeutic target and respective drugs to treat COVID-19. However, Gordon et al., followed a comprehensive approach and cloned, tagged and expressed 26 of the 29 SARS-CoV-2 proteins and identified 332 high-confidence SARS-CoV-2-human protein-protein interactions.[6] Majority of SARS-CoV-2 interacting proteins were associated with replication, epigenetic regulation and vesicle trafficking pathways. [6] In their human lung mRNA expression profile, they identified enrichment of SARS-CoV-2-interacting protein and an epigenetic regulator, histone deacetylase 2 (HDAC2), which regulates epigenetics by removing acetyl groups from histones.[6, 7] Many non-histone proteins such as transcription factors, chaperones and viral proteins are also subjected to acetylation.[7] Gordon et al., also identified 66 therapeutic targets for 69 compounds that includes Valproic Acid (VPA).[6] VPA is a FDA-approved HDAC2 inhibitor drug used to treat central nervous system disease such as epilepsy, and cancer.[8-10] VPA inhibits HDAC2 by inducing its proteasomal degradation. [9]

There are unequivocal evidence that angiotensin-converting enzyme 2 (ACE-2) receptors are the 'entry 
door' for SARS-CoV-2 to infect cells.[11, 12] ACE-2 receptors are mainly expressed on endothelial and epithelial cells. [2, 11] Endothelial cells (ECs) contribute for more than $30 \%$ of all lung cells, $[13]$ and constitute the innermost layer of every blood vessel and respond to constantly varying hemodynamics to maintain homeostasis.[14] ECs are plastic in nature and they have the capability to lose endothelial characteristics and transition into "stem cell-like" mesenchymal cells, this proces is known as endothelial-to-mesenchymal transition (EndMT). [15, 16]

HCQ, which is being tested in various clinical trials against COVID-19, is an autophagy inhibitor.[17] Autophagy is a key homeostatic process, where cytosolic components are degraded and recycled through lysosomes for re-use.[17] We have previously demonstrated that inhibition of endothelial autophagy via genetic deletion of autophagy-related gene 7 (ATG7) or by pharmacologic inhibition with bafilomycin induces EndMT-like phenotypic switching in ECs.[18] Interestingly, a similar effect of VPA was also observed, where VPA induced EndMT-like phenotypic switching in ECs.[19] Given that ACE-2 is expressed basally and widely on the endothelial cells, $[2,11]$ we hypothesized that VPA-induced EndMT-like phenotypic switching cause reduced expression of ACE-2 and thereby would inhibit the SARS-CoV-2 rate of infection. We, for the first time show that VPA down-regulates ACE-2 expression and inhibits the expression of inflammatory cytokines in ECs. We also provide a detailed review on VPA mechanism of action and propose the plausible mechanism of VPA to protect and treat COVID-19 patients encouraging personalized therapy.

\section{Methods}

Human umbilical vein ECs (HUVECs, pooled, Lonza) or human coronary artery EC (HCAECs, Lonza) were cultured in EC growth medium-2 (EGM-2 Bulletkit; Lonza) containing growth factors or MCDB 131 (Gibco) supplemented with serum and antibiotics. Following 60-70\% confluence, cells were starved over-night in 1\% FBS and then treated with 5, 10 and 20mM of VPA (Santa Cruz Biotechnology) for 24 hours. Control group were treated with the diluent. RNA were extracted using Trizol (Invitrogen) and cDNA was synthesized (Quantitect, Qiagen) according to manufacturer's instruction. Quantitative (q)PCR was performed to measure expression level of genes using SYBRß Select Master Mix (Applied Biosystems) and primers for ACE-2[20] IL-6 (Forward - 5'-CATTGGAGCAAGTGTTGGATCTT-3' and Reverse - 5'-GAGCTAATGCATGCCATTCTCA-3'), and inter-cellular adhesion molecule-1 (ICAM-1)[20] in QuantStudio 3 Real-Time PCR System. GAPDH was used as internal control.[20] Differences between two groups were calculated using Student's T-test and differences between more than two groups were calculated using one-way ANOVA with Tukey's test. A p-value of $<0.05$ was considered significant.

\section{Results}

To test our hypothesis, ACE-2 expression was evaluated in cultured HUVECs treated with clinically relevant dose $5 \mathrm{mM}$ of VPA[19, 21] for 24 hours. HUVECs represent the standard cellular model to study endothelial cells in vitro .[19, 20,22] Our qPCR data demonstrated a significant $\sim 70 \%$ reduction in the expression level of ACE-2 in VPA-treated ECs in comparison to control ECs (Fig. 1A ). Given the venous nature of HUVECs, we next evaluated the expression level of ACE-2 in an arterial ECs by treating cultured HCAECs with $5 \mathrm{mM}$ of VPA for 24 hours. Similar to HUVECs, we observed a significant $\sim 70 \%$ reduction in the ACE-2 expression in VPA-treated in comparison to vehicle-treated control HCAECs (Fig. 1B ). To assess dose-response effect of higher dose of VPA on endothelial ACE-2 expression, we treated HUVECs with 10mM and 20mM of VPA for 24 hours and evaluated ACE-2 expression. Our qPCR data showed a significant reduction in ACE-2 expression for both $10 \mathrm{mM}$ and $20 \mathrm{mM}$ of VPA but there was no difference in ACE-2 expression between 10 and $20 \mathrm{mM}$ of VPA treated ECs. Next, we evaluated the effect of VPA on pro-inflammatory IL-6 expression, and endothelial activation marker ICAM-1 expression. We observed a significant down-regulation of IL-6 following $5 \mathrm{mM}$ of VPA treatment to HUVECs in comparison to control (Fig. 1C, D ). Interestingly, VPA also significantly reduced ICAM-1 expression in VPA-treated in comparison to control ECs (Fig. 1E ).

\section{Discussion}

VPA is a well-known HDAC2 inhibitor, which has been safely used for over 50 years as an anti-convulsant drug.[8-10] VPA is taken-up by endothelium immediately within a minute of intravenous injection in hu- 
mans.[23] Cardiovascular complications with wide-spread endothelial dysfunction, thrombosis and endothelitis are rapidly emerging as a key threat in COVID-19 in addition to respiratory disease.[1,2] Endothelium plays a central role in inflammation, thrombosis and cardiovascular complications, and a SARS-CoV-2 use ACE-2 receptors for cell entry, which is mainly expressed on ECs. [2, 11, 12] Altogether, these information indicate for a bigger role for VPA and endothelium in COVID-19. Accordingly, to devise therapeutic strategies to counteract SARS-CoV-2 infection and the associated COVID-19 pathology, it is crucial to understand, and to apply the presented and other published knowledge towards repurposing already approved drugs to treat or prevent COVID-19. Accordingly, we plan to evaluate the role of potential drug VPA on ACE-2 expression in ECs. We also discuss our finding in relation to other published article providing a plausible mechanism for the use of VPA for prevention and treatment of COVID-19 (Fig. 2 ).

Our data demonstrate that VPA treatment to ECs significantly reduced ACE-2 expression. This data is clinically very relevant for COVID-19 as ACE-2 is a cell "entry door" for SARS-Co-2 and also because SARS-CoV-2 infection is shown to be enhanced by over-expression[24] and diminished by inhibition of ACE2. [25-27] The effect of VPA on ACE-2 expression indicates that VPA can inhibit the SARS-CoV-2 rate of infection by reducing its receptor ACE-2 expression level and can be used as a prevention strategy against COVID-19. Next, our data on reduced expression of IL-6 in VPA-treated ECs is also clinically relevant, as the cause of death is the inflammation and thrombosis due to the "cytokine storm" mainly of interleukins such as IL-6 in the lungs of COVID-19 patients.[3] Particularly, level of IL-6 predicts respiratory failure and IL-6 inhibitors are proposed to ameliorate severe lung damage in COVID-19 patients.[28] ECs can secrete pro-inflammatory cytokines and these cytokines "activate" endothelial cells to produce tissue factor, which regulates thrombosis. [29, 30] ICAM-1 is markers for endothelial "activation".[30] Interestingly, VPA also significantly reduced ICAM-1 expression indicating reduced endothelial activation (Fig. 1E ). The presented data is of immense importance as it provides a "treatment" strategy for COVID-19 as VPA-induced downregulation of IL-6 and ICAM-1 are the regulatory molecules implicated in SARS-CoV-2-induced inflammation and coagulation.

PCR array analysis for human endothelial-related genes in VPA-treated ECs is reported with a total 14 significantly up- and 14 down-regulated genes.[19] To evaluate the therapeutic role of VPA in COVID19 , it is important to look at these VPA-induced differentially expressed endothelial genes in relation to inflammation, coagulation, endothelial function, endothelitis, cardiovascular and respiratory diseases. The most up-regulated endothelial genes which are relevant to COVID-19 prevention and/or treatment include BNP (Natriuretic peptide B; 145-fold), MMP-9 (Matrix metallopeptidase 9, 121-fold), PF-4 (Platelet factor 4, 25-fold), T-PA (Plasminogen activator, tissue, 18-fold), COX-2 (Prostaglandin-endoperoxide synthase 2, 3 -fold) and TGF $\beta 1$ (Transforming growth factor, beta 1, 1.3-fold). BNP was the most up-regulated gene in VPA-treated ECs, which is a diagnostic biomarker for cardiac dysfunction, [31] but is also anti-inflammatory and inhibit interleukins. [32, 33] The next up-regulated gene MMP-9 is anti-inflammatory[34, 35] and is associated with lung remodelling. [36] PF-4 is anti-viral as it inhibits human immunodeficiency virus type 1 (HIV-1) infection, [37] and also protects lung against bacterial infection.[38] VPA-induced endothelial and anti-thrombotic T-PA is of particularly relevance to the COVID-19 patients. T-PA treatment is suggested for COVID-19 Associated Acute Respiratory Distress Syndrome (ARDS).[39] Furthermore, VPA is known to have protective effects in severe hemorrhage and ischemia-reperfusion injury.[40] Coagulopathy has become a hallmark of severe COVID-19 with high rates of central line thrombosis and vascular occlusive events (e.g. ischemic limbs, strokes, etc).[39] Ventilator-induced lung injury is another cause in COVID-19 death and T-PA is known to attenuate ventilator-induced lung injury.[41] Overall, VPA-induced T-PA appears to be beneficial to the COVID-19 patients due to its anti-thrombotic activity and attenuation of ventilatorinduced lung injury function. COX-2 was also up-regulated in VPA-treated ECs. Given the important role played by COX-2 in antipyretic nonsteroidal anti-inflammatory drugs (NSAIDs), caution should be taken while prescribing NSAIDs with VPA to COVID-19 patients.[42] The most down-regulated and relevant to COVID-19 prevention and/or treatment genes include TFPI (Tissue factor pathway inhibitor, 6-fold) and SPHK (Sphingosine kinase 1,5-fold). Tissue factor as an initiator of coagulation and inflammation in the lung.[43] Most down-regulated anti-coagulant TFPI activities are reported in the lungs of idiopathic 
pulmonary fibrosis patients, [44] and it is marker for the prediction of deep venous thrombosis and tumor metastasis in patients with lung cancer.[45] Given the increased rate of thrombosis in COVID-19, the status of TFPI needs to be investigated with VPA-treatment. The VPA-induced down-regulation of SPHK1 appears to help COVID-19 treatment as SPHK1 is known to contributes to ventilator-associated lung injury,[46] elevated SPHK1 enhances influenza virus infection,[47] and SPHK1 also serves as a pro-viral factor by regulating viral RNA synthesis and nuclear export of viral ribonucleoprotein complex upon influenza virus infection.[48] VPA-induced down-regulation of SPHK appears to help COVID-19 patients.

The exact mechanism behind HCQ-mediated therapeutic benefit to COVID-19 patients is not completely elucidated but what we know as a fact that HCQ is an autophagy inhibitor.[17] TGF $\beta$-signalling induces EndMT, [16] and loss of endothelial autophagy[18] or VPA-treatment to ECs,[19] both exhibit TGF $\beta$-induced EndMT. It is quite possible that EndMT-associated loss of endothelial function and gain of pro-fibrogenic mesenchymal function is the mechanism behind HCQ-associated benefit in COVID-19 patients. In that scenario, similar benefit can be expected from VPA in COVID-19 patients. EndMT is known to play roles in the development process, $[49]$ and also in diseases such as pulmonary vein stenosis, anomalous vascular remodeling, cerebral cavernous malformations, cancer progression and organ fibrosis. [50, 51] Most importantly, It was reported that $16 \%$ of the lung fibroblasts in pulmonary fibrosis mouse model originated from ECs through EndMT process,[52] and loss of endothelial autophagy-induced EndMT exacerbated lung fibrosis in the same mouse model.[18] Role of EndMT in exacerbation of lung fibrosis might be the reason behind no-effect or adverse effect of HCQ in critically-ill COVID-19 patients, however this needs to be evaluated. Similar, side effect can be expected for VPA, and if true, them precautions should be taken before prescribing VPA to COVID-19 patients with pre-existing pathologies. Furthermore, VPA is an anti-seizure drug and there are reports suggesting that patients with pre-existing seizure disorder may be at greater risk for getting breakthrough seizures. [53] In these COVID-19 patients, VPA can be a preferred treatment option.

This new knowledge about "mechanism of action" of VPA provides a novel potential therapeutic drug target for prevention and treatment of COVID-19, and warrants immediate further investigation in animal models and in humans. We also suggest that VPA based therapeutics may exacerbate EndMT-related pathologies in COVID-19 patients with pre-existing diseases, and promote personalized therapy in such COVID-19 patients. Overall, our manuscript provides a better source of necessary knowledge on VPA for reasearchers and health care practitioners to find treatment for COVID-19. VPA is a FDA approved drugs and any proof of these drugs showing protective effect against COVID-19 will reduce the required drug approval time and it can be fast-tracked for testing against COVID-19.

\section{Acknowledgement}

This work was supported by a grant from the Heart and Stroke Foundation of Canada (G-17-0018688) to KS. KS is also the recipient of the 2018/19 National New Investigator Award- Salary Support from the Heart and Stroke Foundation of Canada, Canada.

\section{Author Contribution Statement}

KS conceived and designed the study. SS helped with data analysis, figures, discussion and manuscript writing.

\section{Conflict of Interest}

None

\section{REFERENCES}

1. Vincent, J.L. and F.S. Taccone, Understanding pathways to death in patients with COVID-19. Lancet Respir Med, 2020. 8 (5): p. 430-432.

2. Varga, Z., et al., Endothelial cell infection and endotheliitis in COVID-19. Lancet, 2020. 395 (10234): p. 1417-1418. 
3. Jose, R.J. and A. Manuel, COVID-19 cytokine storm: the interplay between inflammation and coagulation. Lancet Respir Med, 2020.

4. Choudhary, R., A.K. Sharma, and R. Choudhary, Potential use of hydroxychloroquine, ivermectin and azithromycin drugs in fighting COVID-19: trends, scope and relevance. New Microbes New Infect, 2020: p. 100684.

5. Dong, L., S. Hu, and J. Gao, Discovering drugs to treat coronavirus disease 2019 (COVID-19). Drug Discov Ther, 2020.14 (1): p. 58-60.

6. Gordon, D.E., et al., A SARS-CoV-2 protein interaction map reveals targets for drug repurposing. Nature, 2020.

7. Delcuve, G.P., D.H. Khan, and J.R. Davie, Roles of histone deacetylases in epigenetic regulation: emerging paradigms from studies with inhibitors. Clin Epigenetics, 2012. 4 (1): p. 5.

8. Gottlicher, M., et al., Valproic acid defines a novel class of HDAC inhibitors inducing differentiation of transformed cells. EMBO J, 2001. 20 (24): p. 6969-78.

9. Kramer, O.H., et al., The histone deacetylase inhibitor valproic acid selectively induces proteasomal degradation of HDAC2.EMBO J, 2003. 22 (13): p. 3411-20.

10. Phiel, C.J., et al., Histone deacetylase is a direct target of valproic acid, a potent anticonvulsant, mood stabilizer, and teratogen.J Biol Chem, 2001. 276 (39): p. 36734-41.

11. Hoffmann, M., et al., SARS-CoV-2 Cell Entry Depends on ACE2 and TMPRSS2 and Is Blocked by a Clinically Proven Protease Inhibitor.Cell, 2020. 181 (2): p. 271-280 e8.

12. Walls, A.C., et al., Structure, Function, and Antigenicity of the SARS-CoV-2 Spike Glycoprotein. Cell, 2020. 181 (2): p. 281-292 e6.

13. Crapo, J.D., et al., Cell number and cell characteristics of the normal human lung. Am Rev Respir Dis, 1982. 126 (2): p. 332-7.

14. Deanfield, J.E., J.P. Halcox, and T.J. Rabelink, Endothelial function and dysfunction: testing and clinical relevance. Circulation, 2007. 115 (10): p. 1285-95.

15. Piera-Velazquez, S., Z. Li, and S.A. Jimenez, Role of endothelial-mesenchymal transition (EndoMT) in the pathogenesis of fibrotic disorders. Am J Pathol, 2011. 179 (3): p. 1074-80.

16. van Meeteren, L.A. and P. ten Dijke, Regulation of endothelial cell plasticity by TGF-beta. Cell Tissue Res, 2012. 347 (1): p. 177-86.

17. $\mathrm{Xu}, \mathrm{R}$., et al., The clinical value of using chloroquine or hydroxychloroquine as autophagy inhibitors in the treatment of cancers: A systematic review and meta-analysis. Medicine (Baltimore), 2018.97 (46): p. e12912.

18. Singh, K.K., et al., The essential autophagy gene ATG7 modulates organ fibrosis via regulation of endothelial-to-mesenchymal transition. J Biol Chem, 2015. 290 (5): p. 2547-59.

19. Murugavel, S., et al., Valproic Acid Induces Endothelial-to-Mesenchymal Transition-Like Phenotypic Switching. Front Pharmacol, 2018. 9 : p. 737.

20. Singh, K.K., et al., BRCA1 is a novel target to improve endothelial dysfunction and retard atherosclerosis. J Thorac Cardiovasc Surg, 2013. 146 (4): p. 949-960 e4.

21. Michaelis, M., et al., Valproic acid inhibits angiogenesis in vitro and in vivo. Mol Pharmacol, 2004. 65 (3): p. $520-7$.

22. Singh, S., et al., Endothelial-specific Loss of IFT88 Promotes Endothelial-to-Mesenchymal Transition and Exacerbates Bleomycin-induced Pulmonary Fibrosis. Sci Rep, 2020. 10 (1): p. 4466. 
23. Hammond, E.J., et al., In vivo uptake of valproic acid into brain. Brain Res, 1982. 240 (1): p. 195-8.

24. Yang, X.H., et al., Mice transgenic for human angiotensin-converting enzyme 2 provide a model for SARS coronavirus infection. Comp Med, 2007. 57 (5): p. 450-9.

25. Li, W., et al., Angiotensin-converting enzyme 2 is a functional receptor for the SARS coronavirus. Nature, 2003.426 (6965): p. 450-4.

26. Huentelman, M.J., et al., Structure-based discovery of a novel angiotensin-converting enzyme 2 inhibitor. Hypertension, 2004.44 (6): p. 903-6.

27. Kuba, K., et al., A crucial role of angiotensin converting enzyme 2 (ACE2) in SARS coronavirus-induced lung injury. Nat Med, 2005.11 (8): p. 875-9.

28. Herold, T et al., Level of IL-6 predicts respiratory failure in hospitalized symptomatic COVID-19 patients. doi: https://doi.org/10.1101/2020.04.01.20047381

29. Wu, K.K. and P. Thiagarajan, Role of endothelium in thrombosis and hemostasis. Annu Rev Med, 1996. 47 : p. $315-31$.

30. Videm, V. and M. Albrigtsen, Soluble ICAM-1 and VCAM-1 as markers of endothelial activation. Scand J Immunol, 2008.67 (5): p. 523-31.

31. Corte, T.J., et al., Elevated brain natriuretic peptide predicts mortality in interstitial lung disease. Eur Respir J, 2010.36 (4): p. 819-25.

32. Hu, G., et al., Anti-inflammatory effect of B-type natriuretic peptide postconditioning during myocardial ischemia-reperfusion: involvement of PI3K/Akt signaling pathway. Inflammation, 2014.37 (5): p. $1669-74$.

33. Mezzasoma, L., C. Antognelli, and V.N. Talesa, A Novel Role for Brain Natriuretic Peptide: Inhibition of IL-1beta Secretion via Downregulation of NF-kB/Erk 1/2 and NALP3/ASC/Caspase-1 Activation in Human THP-1 Monocyte. Mediators Inflamm, 2017. 2017 : p. 5858315.

34. Zhang, H., et al., MMP9 protects against LPS-induced inflammation in osteoblasts. Innate Immun, 2020. 26 (4): p. 259-269.

35. Hald, A., et al., MMP9 is protective against lethal inflammatory mass lesions in the mouse colon. Dis Model Mech, 2011.4 (2): p. 212-27.

36. Atkinson, J.J. and R.M. Senior, Matrix metalloproteinase-9 in lung remodeling. Am J Respir Cell Mol Biol, 2003. 28 (1): p. 12-24.

37. Auerbach, D.J., et al., Identification of the platelet-derived chemokine CXCL4/PF-4 as a broad-spectrum HIV-1 inhibitor. Proc Natl Acad Sci U S A, 2012. 109 (24): p. 9569-74.

38. Yue, L., et al., CXCL4 contributes to host defense against acute Pseudomonas aeruginosa lung infection. PLoS One, 2018.13 (10): p. e0205521.

39. Wang, J., et al., Tissue Plasminogen Activator (tPA) Treatment for COVID-19 Associated Acute Respiratory Distress Syndrome (ARDS): A Case Series. J Thromb Haemost, 2020.

40. Causey, M.W., et al., Beneficial effects of histone deacetylase inhibition with severe hemorrhage and ischemia-reperfusion injury. J Surg Res, 2013. 184 (1): p. 533-40.

41. Huang, L.T., et al., Tissue plasminogen activator attenuates ventilator-induced lung injury in rats. Acta Pharmacol Sin, 2012.33 (8): p. 991-7.

42. Simmons, D.L., D. Wagner, and K. Westover, Nonsteroidal anti-inflammatory drugs, acetaminophen, cyclooxygenase 2, and fever.Clin Infect Dis, 2000. 31 Suppl 5 : p. S211-8. 
43. van der Poll, T., Tissue factor as an initiator of coagulation and inflammation in the lung. Crit Care, 2008. 12 Suppl 6 : p. S3.

44. Fujii, M., et al., Relevance of tissue factor and tissue factor pathway inhibitor for hypercoagulable state in the lungs of patients with idiopathic pulmonary fibrosis. Thromb Res, 2000.99 (2): p. 111-7.

45. Fei, X., et al., Tissue Factor Pathway Inhibitor-1 Is a Valuable Marker for the Prediction of Deep Venous Thrombosis and Tumor Metastasis in Patients with Lung Cancer. Biomed Res Int, 2017.2017 : p. 8983763.

46. Wang, Y., et al., Upregulation of sphingosine kinase 1 contributes to ventilator-associated lung injury in a two-hit model.Int J Mol Med, 2019. 44 (6): p. 2077-2090.

47. Carr, J.M., et al., Sphingosine kinase 1 in viral infections.Rev Med Virol, 2013. 23 (2): p. 73-84.

48. Seo, Y.J., et al., Sphingosine kinase 1 serves as a pro-viral factor by regulating viral RNA synthesis and nuclear export of viral ribonucleoprotein complex upon influenza virus infection. PLoS One, 2013. 8 (8): p. e75005.

49. Monaghan, M.G., et al., Endocardial-to-mesenchymal transformation and mesenchymal cell colonization at the onset of human cardiac valve development. Development, 2016. 143 (3): p. 473-82.

50. Piera-Velazquez, S. and S.A. Jimenez, Endothelial to Mesenchymal Transition: Role in Physiology and in the Pathogenesis of Human Diseases. Physiol Rev, 2019. 99 (2): p. 1281-1324.

51. Kato, H., et al., Pulmonary vein stenosis and the pathophysiology of "upstream" pulmonary veins. J Thorac Cardiovasc Surg, 2014. 148 (1): p. 245-53.

52. Hashimoto, N., et al., Endothelial-mesenchymal transition in bleomycin-induced pulmonary fibrosis. Am J Respir Cell Mol Biol, 2010.43 (2): p. 161-72.

53. Seizures and COVID-19. https://www.medpagetoday.com/infectiousdisease/covid19/86118.

Figure legends

Figure 1. VPA-treatment down-regulates ACE-2 and inflammatory molecule expression in ECs. HUVECs or HCAECs were treated with either diluent or 5,10 or $20 \mathrm{mM}$ of VPA and total RNA were extracted 24-hours post-treatment. QPCR data demonstrating reduced ACE-2 expression in VPA-treated (A, C) HUVECs and (B) HCAECs. VPA-treatment (5mM) also reduced IL-6 (D) and ICAM-1(E) expression in HUVECs. $\mathrm{N}=3-4$ in triplicates. ${ }^{*} \mathrm{p}<0.05,{ }^{*} \mathrm{p}<0.01,{ }^{* *} \mathrm{p}<0.001$ vs.corresponding diluent control group.

Figure 2. Schematic representing VPA-associated mechanisms in ECs to prevent and protect against COVID-19. VPA-treatment reduces ACE-2 expression, which is a SARS-CoV-2 receptor on EC. VPA also reduces inflammatory IL-6 expression along with endothelial activation marker ICAM-1, which will reduce thrombosis. VPA-induced T-PA expression will further protect against thrombosis, overall reducing SARS-CoV-2 rate of infection, SARS-CoV-2-induced inflammation and thrombosis to reduce pneumonia, lung failure and deaths in COVID-19 patients.

\section{Hosted file}

VPA_COVID_Figs_9May2020.docx available at https://authorea.com/users/320956/articles/450345valproic-acid-in-prevention-and-treatment-of-covid-19 